\title{
Especies nuevas de Americanura (Collembola: Neanuridae) de América Latina
}

\author{
New species of Americanura (Collembola: Neanuridae) from Latin America
}

\author{
José G. Palacios-Vargas ${ }^{1 *}$, José C. Simón Benito² y Jorge Paniagua Nucamendi1 \\ ${ }^{1}$ Laboratorio de Ecología y Sistemática de Microartrópodos, Facultad de Ciencias, Universidad Nacional Autónoma de México, 04510 México, D.F., \\ México. \\ ${ }^{2}$ Unidad de Zoología, Facultad de Ciencias, Universidad Nacional Autónoma de Madrid, C/Darwin 2, 28049, Canto Blanco, Madrid, España. \\ *Correspondencia: troglolaphysa@hotmail.com
}

\begin{abstract}
Resumen. Se describen e ilustran 5 especies nuevas de Americanura, 3 son de México (estados de Tabasco, Oaxaca y México) y 2 de Panamá (provincia de Colón). Se incluye una clave para todas las especies conocidas del género.

Palabras clave: Americanura, México, Panamá, taxonomía, claves.

Abstract. Five new species of Americanura are described and illustrated, 3 of them from Mexico (Tabasco, Oaxaca and Estado de México) and 2 from Panama (province of Colón). A key for all known species of the genus is included.
\end{abstract}

Key words: Americanura, Mexico, Panama, taxonomy, keys.

\section{Introducción}

Las Neanuridae constituyen la mayor familia de Collembola, en cuanto al número de especies (1372), las que se caracterizan por la ausencia de placa mandibular y presentar la maxila con un cardo largo y delgado entre el estipe y el fulcro. Deharveng (1981) demostró la importancia de la quetotaxia, sobre todo de la parte dorsal del cuerpo y en particular de las antenas, estableciendo el género Sensillanura para incluir ciertas especies de los Estados de Unidos de América y una de los Alpes austriacos, con base en una sensila hipertrofiada del antenito IV. Posteriormente, Cassagnau (1982, 1983, 1988) estudió las relaciones evolutivas de los diferentes grupos de la subfamilia Neanurinae y creó el linaje "Sensillanurina", interesante desde el punto de vista evolutivo de las sedas, ya que algunas de ellas se han desarrollado muy grandes, alargadas, con ciliaciones o barbulaciones, y otras se han vuelto planas y con ornamentaciones que les dan la apariencia de hojas de palma, existiendo además una clara reducción en el número total de sedas dorsales. Actualmente dicho linaje contiene taxones endémicos de la región neotropical e incluye 3 géneros: Sensillanura con 5 especies, Palmanura con 14 y Americanura con 15, más las 5 nuevas que se describen en el presente trabajo.

Recibido: 12 septiembre 2008; aceptado: 10 noviembre 2008

\section{Material y métodos}

Se revisaron ejemplares de Americanura montados en líquido de Hoyer para su estudio. Algunos provienen de muestras recientes (2003) recolectadas en el dosel de la selva alta en la costa atlántica de Panamá dentro del proyecto Investigating the Biodiversity of Soil and Canopy Arthropods (IBISCA) coordinado por Yves Basset del Instituto Smithsonian de Investigaciones Tropicales (STRI, por sus siglas en inglés) y con participación de Rosa Gabriela Castaño Meneses de la Facultad de Ciencias, UNAM. El material proveniente de los estados de México y Tabasco fue obtenido gracias a proyectos realizados en el Laboratorio de Ecología y Sistemática de Microartrópodos, y los de Oaxaca de muestras recolectadas en un proyecto del Laboratorio de Edafología, coordinado por Norma García Calderón, ambos laboratorios de la Facultad de Ciencias, UNAM.

Los dibujos de las especies nuevas se realizaron con ayuda de una cámara translúcida adaptada a un microscopio de constraste de fases marca Carl Zeiss, que a su vez permitió elaborar los cuadros de la quetotaxia y su descripción. La terminología para la descripción de las especies nuevas se basa principalmente en los trabajos de Deharveng (1981), Deharveng y Weiner (1984) y la propuesta por Palacios-Vargas y Simón Benito (2007). Los cuadros de la quetotaxia representan las sedas que tienen por medio segmento. Las abreviaturas para el tipo de sedas 
son: MB (macrosedabarbulada), mb (microseda barbulada), m (microseda), M (macroseda lisa) s, (seda sensorial). Las sedas cefálicas por su posición: Af (antenofrontal), $\mathrm{Cl}$ (clipeal), Oc (ocular), Oca (ocular anterior), Ocm (ocular media), Ocp (ocular posterior). Para los artejos y segmentos: Ant. (artejo antenal), Th. (segmento torácico), Abd. (segmento abdominal). Por su posición los tubérculos son: Di (dorsointerno), De (dorsoexterno), DL (dorso lateral), L (lateral). La sensila de guardia ventral del artejo antenal III: (S.g.v.). Todo el material tipo está en montado en preparaciones permanentes y queda resguardado en la colección del laboratorio de adscripción del primer autor.

\section{Redescripción}

Americanura (modificada de Cassagnau, 1983)

Diagnosis. $2+2$ ojos pigmentados (sólo A. nova carece de ojos). Cuerpo sin pigmento azul hipodérmico. Piezas bucales reducidas, maxilas estiletiformes. Sensila S7 hipertrofiada, cuando menos 2 veces el grosor de las otras. Sedas cefálicas posteriores en 2 grupos, el dorsointerno con una seda (Di1) aislada y el dorsoexterno con las sedas Di2 + De2 + De1 reunidas. De2 está localizada detrás de Di2. El tubérculo dorso lateral (DL) siempre está separado y generalmente con 2 sedas. Tubérculo lateral y subocular $(\mathrm{L}+\mathrm{So})$ fusionados, difíciles de distinguir. Tórax I sin tubérculo Di ni seda, el tubérculo De con 1 o 2 sedas y una seda en el dorso lateral (DL). Abdomen con tubérculo dorsointerno (Di) del cuarto y quinto segmento con 2 o 3 sedas. En el segmento abdominal V, los tubérculos De, DL y L fusionados y con quetotaxia reducida. Con 6 u 8 sedas crenuladas entre las sedas sensoriales del Abd. IV y 4 o 6 entre las del Abd. V. Las sedas de los tubérculos dorsointernos y dorsoexternos crenuladas o barbuladas, rara vez lisas o palmeadas.

Clave para las especies de Americanura

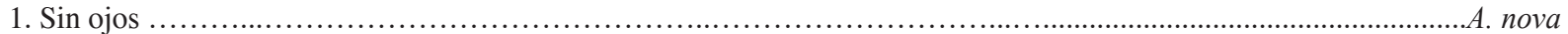

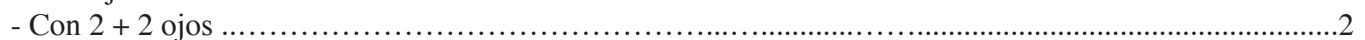

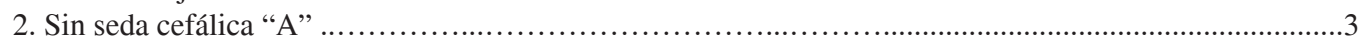

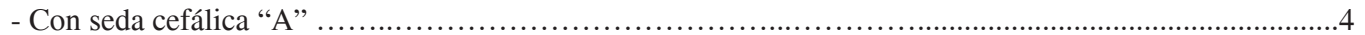

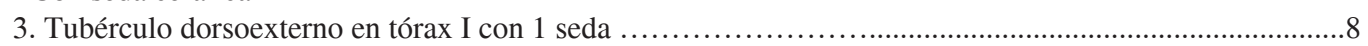

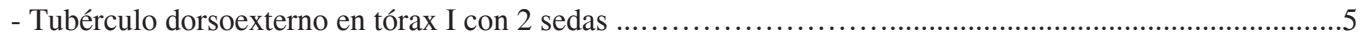

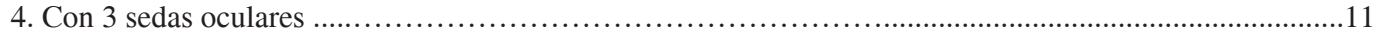

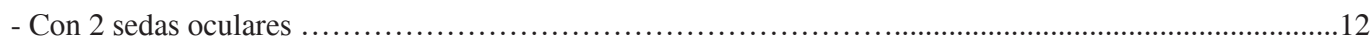

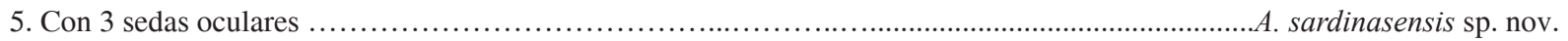

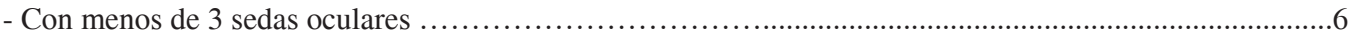

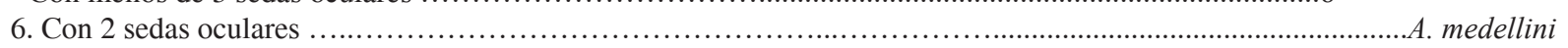

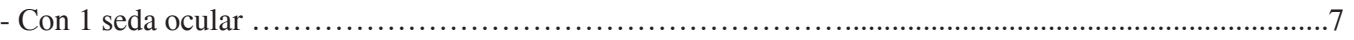

7. Tubérculo cefálico dorsoexterno con 1 seda, tubérculo dorsointerno del tórax II con 2 sedas

- Tubérculo cefálico dorsoexterno con 3 sedas, tubérculo dorsointerno del tórax II con 3 sedas ....... A. izabalana

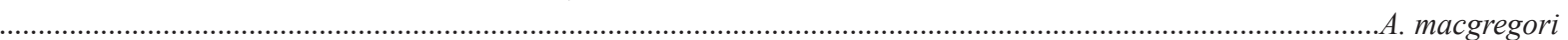

8. Tubérculo cefálico dorsoexterno con 3 sedas, 3 sedas oculares, sedas del cuerpo lisas.

A. bara

- Tubérculo cefálico dorsoexterno con 1 seda, menos de 3 sedas oculares, sedas del cuerpo barbuladas o palmeadas .9

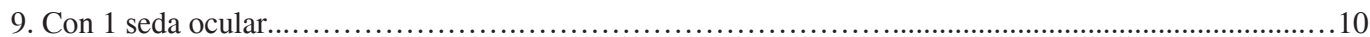

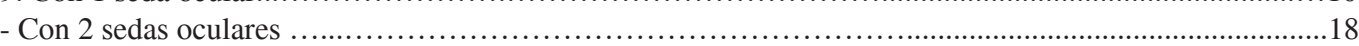

10. Tubérculo dorsointerno del segmento abdominal V con 3 sedas, tubérculo dorso lateral del tórax II y III con 2 sedas, sedas del cuerpo barbuladas.

- Tubérculo dorsointerno del segmento abdominal V con 1 seda, tubérculo dorso lateral del

tórax II y III con 1 seda, sedas del cuerpo palmeadas ......................................................................... guatemalteca

11. Tubérculo cefálico dorsoexterno con 2 sedas, uña con 1 diente grueso

interno. A. unguimitl

- Tubérculo cefálico dorsoexterno con 3 sedas, uña sin diente grueso interno

12. Tubérculo dorsoexterno sobre el tórax I con 2 sedas, tubérculo dorsointerno del tórax II

y III con 3 sedas $\ldots \ldots \ldots \ldots \ldots \ldots \ldots \ldots \ldots \ldots \ldots \ldots \ldots \ldots \ldots \ldots \ldots \ldots \ldots \ldots \ldots \ldots \ldots \ldots \ldots \ldots \ldots \ldots \ldots \ldots \ldots \ldots \ldots \ldots \ldots$
- Tubérculos dorsoexternos del tórax I con 1 seda, tubérculo dorsointerno del tórax II y III

con menos de 3 sedas

A. castillorum 
13. Tubérculo dorsointerno del tórax II al segmento abdominal III con 1 seda 14

- Tubérculo dorsointerno del tórax II al segmento abdominal III con 2 sedas .............................. janssensi sp. nov.

14. Segmento abdominal IV con 4 tubérculos, tubérculo lateral de Abd. II y III con 2 sedas .....

A. banksi

- Segmento abdominal IV con 3 tubérculos, tubérculo lateral de Abd. II y III con 3 sedas

15. Todas las sedas del cuerpo lisas, sedas oculares Oca y Ocp son macrosedas lisas, Ocm

. es macroseda lisa.

- La mayoría de las sedas del cuerpo barbuladas, por lo menos 1 seda ocular es barbulada

16. Tubérculo dorsoexterno de Abd. IV con 2 sedas, tubérculo dorsointerno de Abd. V

con 2 sedas.

- Tubérculo dorsoexterno de Abd. IV con 3 sedas, tubérculo dorsointerno de Abd. V

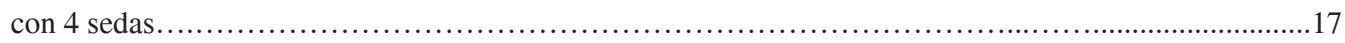

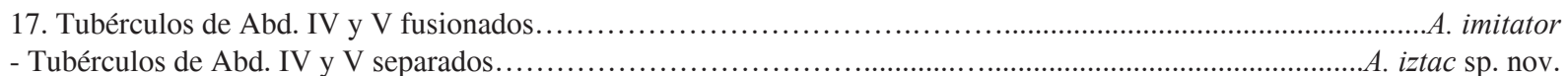

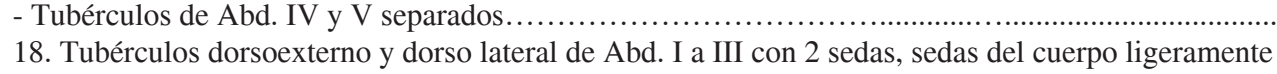

barbuladas..

A. basseti sp. nov.

- Tubérculos dorsoexterno y dorso lateral de Abd. I a III con 1 seda, sedas del cuerpo claramente palmeadas o

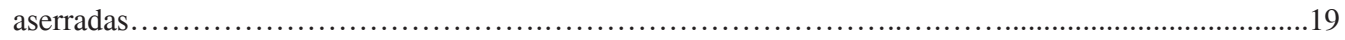

19. Tubérculo dorso lateral de Th. II y III con 1 seda, tubérculo lateral de Abd. I a III

con 2 sedas, la mayoría de sedas dorsales palmeadas....

A. setafoliacea

- Tubérculo dorso lateral de Th. II y III con 2 sedas, tubérculo lateral de Abd. I a III

con 3 sedas, la mayoría de sedas dorsales aserradas. .

A. sotanophila

\section{Americanura sardinasensis sp. nov. (Figs. 1-7)}

Longitud de adultos $(\mathrm{n}=7) 1.10 \mathrm{~mm}$ y juveniles $(\mathrm{n}=6)$ $0.82 \mathrm{~mm}$. De color blanco. Granulación cuticular fuerte, casi del tamaño de un ojo. Tubérculos bien desarrollados. Con 4 tipos de sedas corporales, macrosedas barbuladas (MB) $60 \mu \mathrm{m}(25-87.5 \mu \mathrm{m})$, microsedas barbuladas (mb) $20 \mu \mathrm{m}(17.5-20 \mu \mathrm{m})$, microsedas lisas (m) $17.5 \mu \mathrm{m}$ (7.5 - $30 \mu \mathrm{m})$ y macrosedas lisas (M) $52.5 \mu \mathrm{m}(50-55 \mu \mathrm{m})$ con pocas barbulaciones, además de las sedas sensoriales (ss) $56.1 \mu \mathrm{m}(25-100 \mu \mathrm{m})$. Ant. I con 7 sedas, 3 son macrosedas barbuladas. Ant. II con 11 sedas, una de ellas ligeramente barbulada. Órgano sensorial del Ant. III con 2 microsensilas globulares bajo un repliegue tegumentario y 2 sensilas de guardia, la sensila de guardia ventral (sgv) es ligeramente curva y hay 1 microsensila ventro externa. Ant. IV de forma típica para el género con la sensila S7 hipertrofiada. Organito subapical ausente. Con $2+2$ ojos con pigmento negro. Mandíbulas con 3 dientes (Fig. 6), maxilas estiliformes (Fig. 5). Cabeza con los tubérculos oculares dorsointernos (Di) y dorsoexternos (De) bien desarrollados. Quetotaxia cefálica (Cuadro 1) como se muestra en la figura 1. Tibiotarsos I, II y III sin tenent hairs, con 18, 18 y 17 sedas respectivamente (Fig. 4). Sin apéndice empodial. La quetotaxia torácica y abdominal se ilustra en las figuras 1 y 2 y la quetotaxia total se muestra en el Cuadro 1 . Tubo ventral con $4+4$ sedas, las 2 distales subiguales, las basales son diferentes, una de ellas más larga.

\section{Resumen taxonómico}

Material tipo. Holotipo hembra preadulta, 1 paratipo hembra, 5 paratipos machos, 6 paratipos juveniles. Localidad Tipo. MÉXICO: Estado de Tabasco: Cueva de las Sardinas, cámara tercera, 02-VII-2001, hojarasca; 2 paratipos de la misma localidad, segunda cámara, 12.XI.1998, hojarasca, D. Estrada col.

Etimología. La especie se nombra por la localidad tipo, la Cueva de Las Sardinas.

\section{Comentarios taxonómicos}

Americaura sardinasensis sp. nov. es cercana a $A$. unguimitl y A. nova. Las diferencias entre estas especies se muestran en el Cuadro 2. La ausencia de la seda cefálica A es el carácter más notorio de la nueva especie, ya que se encuentra en $A$. unguimitl, así como la diferente quetotaxia en los tubérculos dorsoexternos. Por su parte $A$. nova carece de ojos.

Variación intraespecífica. Presencia de algunos casos de sedas supernumerarias. En la cabeza de un ejemplar había 1 seda G extra. Otro ejemplar tenía 4 sedas en un tubérculo dorsointerno en ambos lados, la seda supernumeraria es una microseda. En el segmento abdominal I del lado izquierdo hay 1 tubérculo dorsointerno con 1 microseda extra y del derecho hay sólo 2. En los tubérculos Di de los 

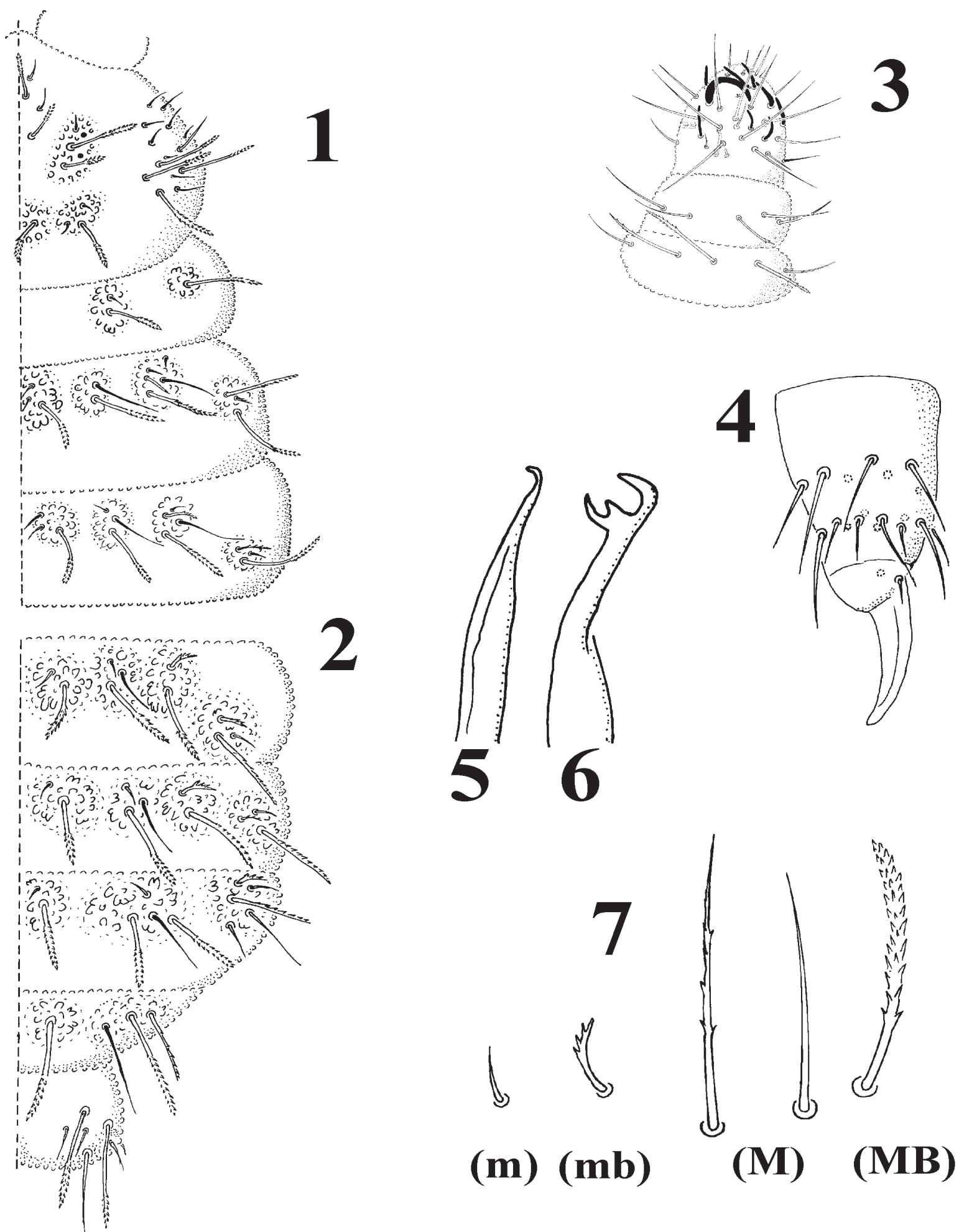

Figs. 1-7. Americanura sardinasensis sp. nov. 1, quetotaxia dorsal de la cabeza y segmentos torácicos I-III; 2 , quetotaxia abdominal de los segmentos II-VI; 3, quetotaxia dorsal de antenitos I-IV, sensila hipertrofiada S7 en Ant.4; 4, tibiotarso; 5, maxila; 6, mandíbula; 7 , tipos de seda, de izquierda a derecha: microseda $(\mathrm{m})$, microseda barbulada $(\mathrm{mb})$, macroseda $(\mathrm{M})$, macroseda barbulada (MB). 
Cuadro 1. Quetotaxia total de Americanura sardinasensis sp. nov.

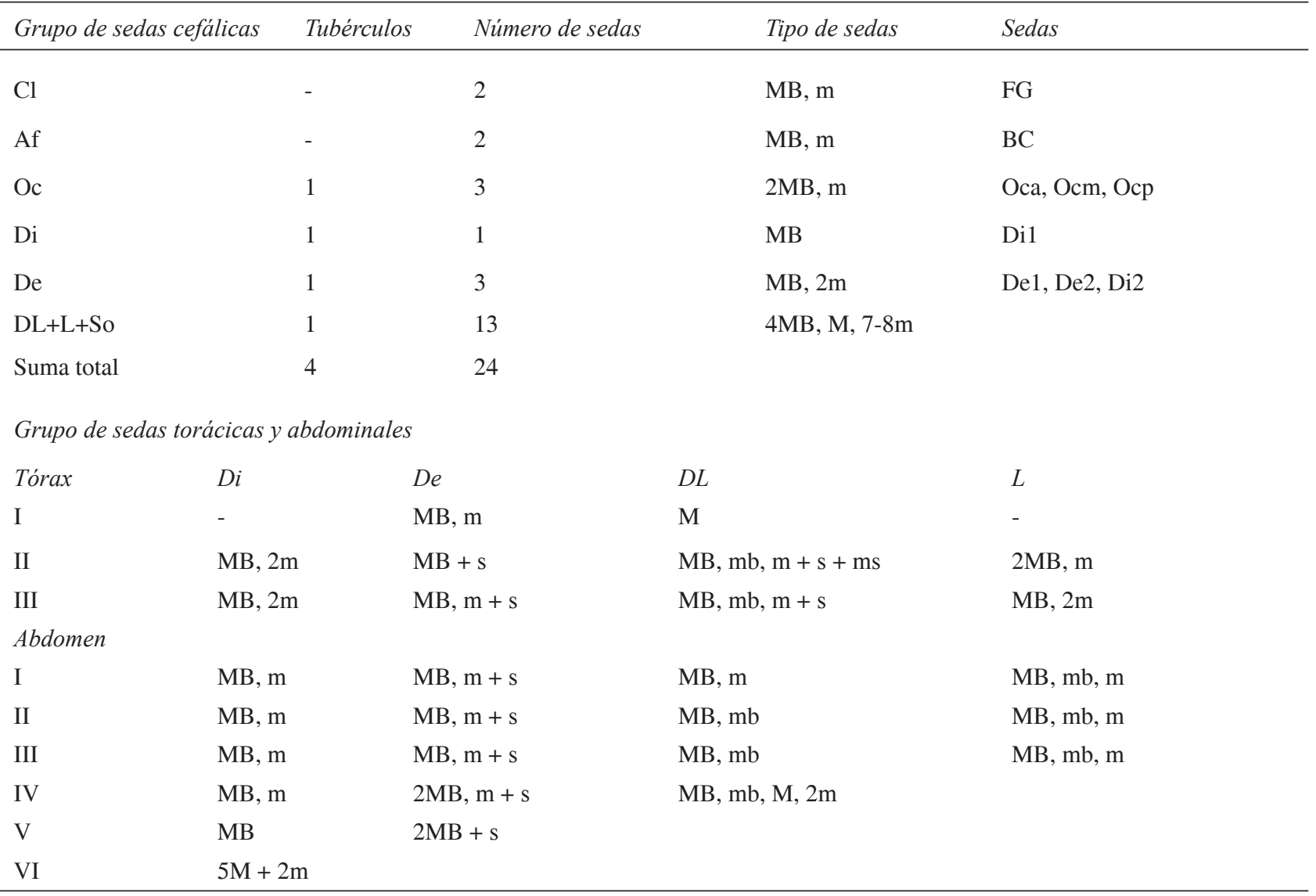

Cuadro 2. Comparación de Americanura sardinasensis sp. nov. y las especies más cercanas

\begin{tabular}{llllll}
\hline Especie & Cabeza & Di & De & DL & $L$ \\
\hline sardinasensis sp. nov. & B, C, F, G & $-33 / 222217$ & $222 / 22232$ & $133 / 2225$ & $-43 / 333$ \\
unguimitl & A, B, C, F, G & $-33 / 222227$ & $222 / 22232$ & $133 / 2223$ & $-43 / 3(3-4) 4$ \\
nova & A, C, F, G & $-33 / 222257$ & $222 / 222$ & $133 / 222$ & $133 / 333$ \\
\hline
\end{tabular}

segmentos torácicos I y II una de las 2 microsedas puede ser barbulada.

\section{Americanura janssensi sp. nov. (Figs. 8-9)}

Longitud $(\mathrm{n}=2)$ adulto $0.90 \mathrm{~mm}$ y juvenil $(\mathrm{n}=1) 0.92 \mathrm{~mm}$. Color blanco. Granulación cuticular mediana, la mitad de una corneola. Tubérculos bien desarrollados. Con 4 tipos de sedas corporales, macrosedas densamente barbuladas (MB) $105 \mu \mathrm{m}(50-147.5 \mu \mathrm{m})$, microsedas barbuladas (mb) $35 \mu \mathrm{m}(22.5$ - $42.5 \mu \mathrm{m})$, microsedas lisas (m) 15.0 $\mu \mathrm{m}$ y macrosedas lisas (M) $37.5 \mu \mathrm{m}$, además de las sedas sensoriales (ss) $63.9 \mu \mathrm{m}(45-87.5 \mu \mathrm{m})$. Ant. I con 7 sedas,
3 son macrosedas largas muy barbuladas. Ant. II con 11 sedas, una de ellas claramente barbulada. Órgano sensorial del Ant. III con 2 microsensilas globulares bajo un repliegue tegumentario y 2 sensilas de guardia, la sensila de guardia ventral (sgv) es casi recta y existe una microsensila ventro externa. Ant. IV de forma típica para el género con la sensila S7 hipertrofiada. Organito subapical ausente. Con $2+2$ ojos con pigmento negro. Mandíbulas con 3 dientes y maxilas estiletiformes. Cabeza con los tubérculos cefálicos, ocular, dorsointerno (Di), antenofrontal y clipeal, y dorsoexterno (De) bien desarrollados. Quetotaxia cefálica (Cuadro 3) ilustrada en la Fig. 8. Tibiotarsos I, II y III sin tenent hairs, con 18, 18 y 17 sedas respectivamente. Sin 


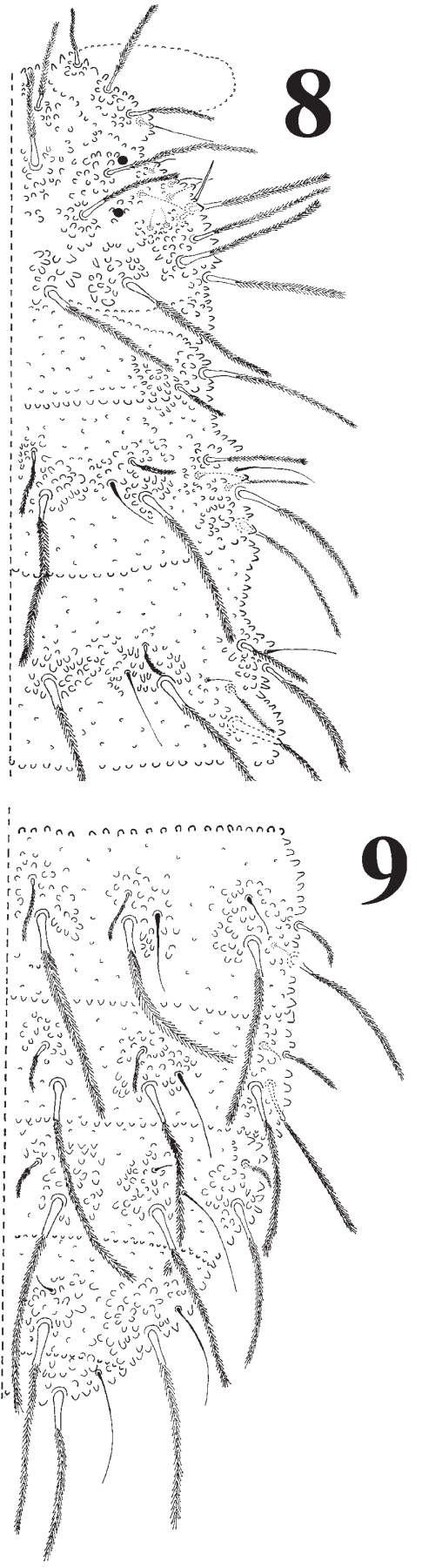

apéndice empodial. La quetotaxia torácica y abdominal se muestra en las figuras 8 y 9. La quetotaxia total se muestra en el Cuadro 3. Tubo ventral con $4+4$ sedas basales, 3 pares dispuestos longitudinalmente subiguales, el par distal es más largo.
Figs. 8-11; 8 y 9, Americanura janssensi sp. nov. 8, quetotaxia dorsal de la cabeza y segmentos torácicos I-III; 9, quetotaxia abdominal de los segmentos II-VI. 10 y 11, Americanura iztac sp. nov.; 10, quetotaxia dorsal de la cabeza y segmentos torácicos I-III; 11, quetotaxia abdominal de los segmentos I-VI.

\section{Resumen taxonómico}

Material tipo. Holotipo hembra y 2 paratipos preadultos. Localidad tipo. MÉXICO: Oaxaca, Santa Cruz (Ixtlán de Juárez). Holotipo de $1400 \mathrm{~m}$ altitud, suelo Podzol, paratipos de suelo a $2550 \mathrm{~m}$ de altitud, Mayo 2006, N. García Calderón col.

Etimología. Esta especie se nomina en honor a Frans Janssens, por su gran labor en la creación de la página de los colémbolos del mundo (http://www.geocities. com/fransjanssens/ index.html).

\section{Comentarios taxonómicos}

Americanura janssensi sp. nov. es similar a $A$. izabalana; sin embargo, difiere en que $A$. izabalana carece de la seda cefálica A. Por otro lado, el tubérculo dorsoexterno del tórax de $A$. janssensi sp. nov. sólo tiene 1 seda, mientras que $A$. izabalana tiene 2. Los tubérculos dorsointernos del tórax III y Ab. I de la nueva especie sólo presenta 2 sedas, mientras que en $A$. izabalana presenta 3 (Cuadro 4). 
Cuadro 3. Quetotaxia total de Americanura janssensi sp. nov.

\begin{tabular}{|c|c|c|c|c|c|c|}
\hline \multicolumn{2}{|c|}{ Grupo de sedas cefálicas } & \multicolumn{2}{|l|}{ Tubérculos } & Número de sedas & Tipo de sedas & \multirow{2}{*}{$\frac{\text { Sedas }}{\mathrm{F}, \mathrm{G}}$} \\
\hline $\mathrm{Cl}$ & & 1 & & 2 & $\mathrm{MB}, \mathrm{m}$ & \\
\hline Af & & 1 & & 2 & $\mathrm{MB}, \mathrm{mb}$ & A, B \\
\hline Oc & & 1 & & 2 & $2 \mathrm{MB}$ & Ocm, Ocp \\
\hline Di & & 1 & & 1 & MB & Di1 \\
\hline $\mathrm{De}$ & & 1 & & 1 & MB & De1 \\
\hline $\mathrm{DL}+\mathrm{L}+\mathrm{So}$ & & 3 & & 11 & $4 \mathrm{MB}, \mathrm{M}, 6 \mathrm{~m}$ & \\
\hline Suma total & & 8 & & 19 & & \\
\hline \multicolumn{7}{|c|}{ Grupo de sedas torácicas y abdominales } \\
\hline Tórax & $D i$ & & $D e$ & & $D L$ & $L$ \\
\hline I & - & & $\mathrm{mb}, \mathrm{m}$ & & MB & - \\
\hline II & $\mathrm{MB}, \mathrm{mb}$ & & $\mathrm{MB}, \mathrm{mb}+$ & $+\mathrm{s}$ & $2 \mathrm{MB}+\mathrm{s}$ & $3 \mathrm{MB}, \mathrm{m}$ \\
\hline III & $\mathrm{MB}, \mathrm{mb}$ & & $\mathrm{MB}, \mathrm{mb}+$ & $+s$ & $2 \mathrm{MB}, \mathrm{mb}+\mathrm{s}$ & $2 \mathrm{MB}, \mathrm{m}$ \\
\hline \multicolumn{7}{|l|}{ Abdomen } \\
\hline I & $\mathrm{MB}, \mathrm{mb}$ & & $\mathrm{MB}, \mathrm{mb}+$ & $+\mathrm{s}$ & $\mathrm{MB}, \mathrm{m}+\mathrm{s}$ & $\mathrm{MB}, \mathrm{mb}, \mathrm{m}$ \\
\hline II & $\mathrm{MB}, \mathrm{mb}$ & & $\mathrm{MB}, \mathrm{mb}+$ & $+s$ & $\mathrm{MB}, \mathrm{m}+\mathrm{s}$ & $\mathrm{MB}, \mathrm{mb}, \mathrm{m}$ \\
\hline III & $\mathrm{MB}, \mathrm{mb}$ & & $\mathrm{MB}, \mathrm{mb}+$ & $+s$ & $2 \mathrm{MB}$ & $\mathrm{MB}, \mathrm{mb}$ \\
\hline IV & $\mathrm{MB}, \mathrm{mb}$ & & $\mathrm{MB}, \mathrm{m}+$ & & $\mathrm{MB}, \mathrm{mb}$ & \\
\hline V & $\mathrm{MB}, \mathrm{mb}$ & & $\mathrm{MB}+\mathrm{s}$ & & & \\
\hline VI & $\mathrm{MB}+\mathrm{s}$ & & & & & \\
\hline
\end{tabular}

Cuadro 4. Comparación entre Americanura janssensi sp. nov. y las especies más cercanas

\begin{tabular}{llllll}
\hline Especie & Cabeza & Di & De & DL & $L$ \\
\hline janssensi sp nov. & $\mathrm{A}, \mathrm{B}, \mathrm{F}, \mathrm{G}$ & $-22 / 222221$ & $222 / 22221$ & $123 / 2222$ & $-43 / 332$ \\
castillorum & $\mathrm{A}, \mathrm{B}, \mathrm{F}, \mathrm{G}$ & $-33 / 222227$ & $233 / 33333$ & $154 / 2222$ & $-33 / 3333$ \\
banksi & $\mathrm{A}, \mathrm{B}, \mathrm{F}, \mathrm{G}$ & $-11 / 222222$ & $-11 / 11111$ & $122 / 1111$ & $133 / 2334$ \\
izabalana & $\mathrm{B}, \mathrm{F}, \mathrm{G}$ & $-22 / 22227$ & $232 / 22275$ & $133 / 222$ & $-32 / 333$ \\
\hline
\end{tabular}

Variación intraespecifica. Se compararon 2 ejemplares preadultos (hembra) y 1 juvenil. En los 3 organismos, las microsedas de los tubérculos L+So varían entre 4 y 5 , sólo en el juvenil se observó que en el DL, a diferencia de los otros (2MB, mb) presenta una macroseda barbulada más. A partir de la quetotaxia patrón $(2 \mathrm{MB}, \mathrm{mb})$ para los segmentos torácicos II y III se observó en los preadultos una macroseda barbulada y 2 microsedas barbuladas, y sólo uno de ellos repetía el mismo patrón en el segmento III. En el tubérculo Di del segmento abdominal IV puede haber una microseda barbulada o no, lo mismo que en el tubérculo dorsoexterno (De) de los segmentos II, III y IV.
Sólo un organismo presentó 2 microsedas en el tubérculo L del segmento abdominal IV. Existe una variación significativa en el Ab. VI y es que puede haber de 1 a 3 sedas macrobarbuladas, 2 o 3 macrosedas y de 2 a 4 microsedas.

Americanura iztac sp. nov. (Figs. 10-11)

Longitud de adultos $(\mathrm{n}=6) 1.59 \mathrm{~mm}$. Color blanco. Granulación cuticular moderada. Todos los tubérculos bien desarrollados. Con 4 tipos de sedas corporales, macrosedas barbuladas $(\mathrm{MB}) 107 \mu \mathrm{m}(75-147.5 \mu \mathrm{m})$, microsedas 
Cuadro 5. Quetotaxia total de Americanura iztac sp. nov.

\begin{tabular}{|c|c|c|c|c|c|c|}
\hline \multicolumn{2}{|c|}{ Grupo de sedas cefálicas } & \multicolumn{2}{|l|}{ Tubérculos } & Número de sedas & Tipo de sedas & \multirow{2}{*}{$\frac{\text { Sedas }}{\mathrm{D}, \mathrm{F}, \mathrm{G}}$} \\
\hline $\mathrm{Cl}$ & & 1 & & 3 & $\mathrm{MB}, \mathrm{mb}, \mathrm{m}$ & \\
\hline Af & & 1 & & 1 & MB & $\mathrm{C}$ \\
\hline Oc & & 1 & & 2 & $\mathrm{MB}, \mathrm{mb}$ & Ocm, Ocp \\
\hline Di & & 1 & & 1 & $\mathrm{MB}, \mathrm{mb}$ & Di1 \\
\hline De & & 1 & & 3 & $2 \mathrm{MB}, \mathrm{mb}$ & De1, De2, Di2 \\
\hline $\mathrm{DL}+\mathrm{L}+\mathrm{So}$ & & 1 & & 7 & $\mathrm{MB}, \mathrm{mb}$ & \\
\hline Suma total & & 6 & & 17 & & \\
\hline \multicolumn{7}{|c|}{ Grupo de sedas torácicas y abdominales } \\
\hline Tórax & $D i$ & & $D e$ & $D L$ & & $L$ \\
\hline I & - & & $\mathrm{MB}, \mathrm{mb}$ & MB & & - \\
\hline II & $\mathrm{MB}, 2 \mathrm{mb}$ & & $\mathrm{MB}, \mathrm{mb}+\mathrm{s}$ & MB & $2 m b+s$ & $2 \mathrm{MB}$ \\
\hline III & $\mathrm{MB}, 2 \mathrm{mb}$ & & $\mathrm{MB}, \mathrm{mb}+\mathrm{s}$ & MB & $2 m b+s$ & $\mathrm{MB}, 2 \mathrm{mb}$ \\
\hline \multicolumn{7}{|l|}{ Abdomen } \\
\hline I & $\mathrm{MB}, \mathrm{mb}$ & & $\mathrm{MB}, \mathrm{mb}+\mathrm{s}$ & MB & $2 m b+s$ & $2 \mathrm{MB}, \mathrm{m}$ \\
\hline II & $\mathrm{MB}, \mathrm{mb}$ & & $2 \mathrm{MB}, \mathrm{mb}+\mathrm{s}$ & $\mathrm{MB}$ & & $\mathrm{MB}, \mathrm{mb}$ \\
\hline III & $\mathrm{MB}, \mathrm{mb}$ & & $\mathrm{Mb}+\mathrm{s}$ & MB & & $\mathrm{MB}, \mathrm{mb}$ \\
\hline IV & $\mathrm{MB}, \mathrm{mb}$ & & $\mathrm{MB}, \mathrm{mb}+\mathrm{s}$ & $\mathrm{MB}$ & & $\mathrm{mb}, \mathrm{m}, \mathrm{M}$ \\
\hline V & $\mathrm{MB}, \mathrm{mb}$ & & $2 \mathrm{MB}, \mathrm{mb}+\mathrm{s}$ & $\mathrm{MB}$ & $2 \mathrm{M}, 2 \mathrm{mb}$ & \\
\hline VI & $5 \mathrm{MB}, 2 \mathrm{M}$ & & & & & \\
\hline
\end{tabular}

barbuladas (mb) $45.0 \mu \mathrm{m}(17.5$ - $65 \mu \mathrm{m})$, microsedas lisas (m) $30 \mu \mathrm{m}(17.5-37.5 \mu \mathrm{m})$, macrosedas lisas (M) $65 \mu \mathrm{m}$ (45 - $82.5 \mu \mathrm{m})$ con poca barbulación, además de las sedas sensoriales (ss) $41.7 \mu \mathrm{m}(37.5-45 \mu \mathrm{m})$. Ant. I con 7 sedas, 3 son macrosedas barbuladas; Ant. II con 11 sedas, una de ellas ligeramente barbulada. Órgano sensorial del Ant. III con 2 sensilas globulares bajo un repliegue tegumentario y 2 sensilas de guardia, la sensila de guardia ventral (sgv) es ligeramente curva y con 1 microsensila ventro externa. Ant. IV de forma típica para el género con la sensila S7 hipertrofiada. Organito subapical ausente. Con $2+2$ ojos con pigmento negro. Mandíbulas con 3 dientes, maxilas estiletiformes. Cabeza con los tubérculos cefálicos ocular, dorsointerno (Di) y dorsoexterno (De) bien desarrollados. Quetotaxia cefálica (Cuadro 5) ilustrada en la Fig. 10. Tibiotarsos I, II y III sin tenent hairs con 18, 18 y 17 sedas respectivamente. Sin apéndice empodial. La quetotaxia torácica y abdominal se ilustran en las figuras 10 y 11. La quetotaxia total se muestra en el Cuadro 5. Tubo ventral con $4+4$ sedas basales, 3 pares dispuestos longitudinalmente subiguales al par distal que es más largo.

\section{Resumen taxonómico}

Material tipo. Holotipo macho, 2 paratipos hembras, un paratipo macho, 2 paratipos machos preadultos. Localidad tipo. MÉXICO: Estado de México, Vertiente noroeste del volcán Iztaccihuatl. Desde los 3250 hasta los 3440 m de altitud, en musgo arborícola y suelo, 9.XI.2003, 5.VI.2004 y 27.VIII.2005, A. Bernal, L. Cutz y A. García cols.

Etimología. En náhuatl iztac significa blanco, y también se usa como abreviatura del nombre de la localidad tipo

\section{Comentarios taxonómicos}

La quetotaxia de Americanura iztac sp. nov. y la de $A$. imitator son muy similares. La diferencia estriba en los tubérculos laterales del tórax II de $A$. iztac sp. nov., 4 sedas en la nueva especie en lugar de 3 y el tubérculo dorsointerno del segmento abdominal $\mathrm{V}$ con 5 sedas en lugar de 4 (Cuadro 6).

Variación intraespecífica. En la cabeza, en el grupo de sedas Oc puede haber 2 o 3, en el caso de ser 2, ambas son barbuladas, una es macro y la otra micro; en el caso de 3 , hay 1 macroseda barbulada y 2 microsedas no barbuladas. En el conjunto de sedas L+So, 4 de 6 organismos presentaron 1seda macrobarbulada y 6 microsedas (MB, $6 \mathrm{~m})$. En uno de los 2 ejemplares restantes fue posible apreciar $2 \mathrm{MB}, \mathrm{M}, 7 \mathrm{~m}$ y en el otro $2 \mathrm{M}, 7 \mathrm{~m}$. La variación en el tórax se aprecia en el tubérculo lateral de segmento III, 
Cuadro 6. Comparación entre 3 especies de Americanura más cercanas

\begin{tabular}{llllll}
\hline Especie & Cabeza & Di & De & $D L$ & $L$ \\
\hline iztac sp nov. & $\mathrm{C}, \mathrm{D}, \mathrm{F}, \mathrm{G}$ & $-33 / 222227$ & $222 / 23133$ & $-33 / 32225$ & $123 / 3223$ \\
imitator & $\mathrm{A}, \mathrm{B}, \mathrm{D}, \mathrm{F}, \mathrm{G}$ & $-33 / 222247$ & $222 / 2223$ & $133 / 222$ & $-33 / 3335$ \\
basseti sp. nov. & $\mathrm{B}, \mathrm{F}, \mathrm{G}$ & $-11 / 1111-$ & $112 / 22223$ & $133 / 2$ & $-11 / 2353$ \\
\hline
\end{tabular}

donde las 3 sedas presentes pueden ser macrobarbuladas, o 2 macrobarbuladas y 1 microseda, o 1 de cada una, como sigue: MB, mb, m. En el abdomen, 5 de 6 organismos presentaron los tubérculos Di del segmento IV unidos pero no fusionados, sólo en uno se observó un inicio de fusión; en el tubérculo DL del mismo segmento abdominal, 5 de 6 organismos presentaron 5 sedas con la siguiente composición: $2 \mathrm{MB} \mathrm{Mb} \mathrm{M} \mathrm{m} \mathrm{(=5);} 3 \mathrm{MB} 2 \mathrm{mb}(=5) ; 3 \mathrm{MB}$ $2 \mathrm{mb}(=5)$; $\mathrm{MB} 2 \mathrm{~m}(=3) ; \mathrm{MB}$ mb M 2m (=5); MB 2mb M $\mathrm{m}(=5)$. En el segmento abdominal VI, en 5 ejemplares se encontraron 7 sedas con la siguiente variación: $4 \mathrm{MB}$ - M 2m (=7); 2MB - 3M 2m (=7); $2 \mathrm{MB}$ - 3M 2m (=7); $2 \mathrm{MB}$ mb 2M 2m (=7); MB mb 3M 2m(=7) y en el otro organismo se encontraron 6: $\mathrm{MB} \mathrm{mb} 3 \mathrm{M} \mathrm{m}$.

\section{Americanura castagnorum sp. nov. (Figs. 12-13)}

Longitud $(\mathrm{n}=3)$ adultos $0.83 \mathrm{~mm}$ y $(\mathrm{n}=1)$ juvenil $0.37 \mathrm{~mm}$. Color blanco. Granulación cuticular fuerte. Tubérculos bien desarrollados. Con 4 tipos de sedas corporales, macrosedas barbuladas (MB) $72.5 \mu \mathrm{m}(52.5$ - $92.5 \mu \mathrm{m})$, microsedas barbuladas (mb) $47.5 \mu \mathrm{m}$ (25 $42.5 \mu \mathrm{m})$, microsedas lisas (m) $22.5 \mu \mathrm{m}$ (12.5 - $37.5 \mu \mathrm{m})$, macrosedas lisas (M) $57.5 \mu \mathrm{m}(50$ - $67.5 \mu \mathrm{m})$, además de las sedas sensoriales (ss) $58.6 \mu \mathrm{m}(39.5-78 \mu \mathrm{m})$. Ant. I con 7 sedas, 3 son macrosedas barbuladas. Ant. II con 9 sedas, 5 de ellas son macrosedas. Órgano sensorial del Ant. III con 2 sensilas globulares bajo un repliegue tegumentario, y 2 sensilas de guardia, la sensila de guardia ventral (sgv) ligeramente curva. Ant. IV de forma típica para el género con la sensila S7 hipertrofiada. Organito subapical ausente. Con $2+2$ ojos con pigmento negro. Mandíbulas con 3 dientes, maxilas estiletiformes. Cabeza con los tubérculos oculares, dorsointerno (Di) y dorsoexterno (De) bien desarrollados. Quetotaxia cefálica (Cuadro 7), ilustrada en la Fig. 12. Tibiotarsos I, II y III sin tenent hairs, con 18, 18 y 17 sedas respectivamente (Fig. 4). Sin apéndice empodial. La quetotaxia torácica y la abdominal se muestran en las figuras 12 y 13, la quetotaxia total en el Cuadro 7. Tubo ventral con $4+4$ sedas basales, 3 pares dispuestos longitudinalmente subiguales al par distal que son más largas.

\section{Resumen taxonómico}

Material tipo. Holotipo macho, 1 paratipo preadulto macho y un paratipo juvenil. Localidad tipo. PANAMÁ: Provincia de Colón, San Lorenzo, bosque, 8,X,2003; ex suelo, N. Winchester y K. Jordan cols.

Etimología. Esta especie se dedica a Rosa Gabriela Castaño Meneses y Víctor Manuel Castaño Meneses.

\section{Comentarios taxonómicos}

Americanura castagnorum sp. nov. es similar a $A$. guatemalteca, sin embargo, todas las sedas de la primera son barbuladas; y en la segunda, las sedas cefálicas B, F, la de los tubérculos Di, De, así como las Di del tórax II y III son claramente palmeadas, si bien, las demás sedas son barbuladas, son mucho más delgadas que en $A$. castagnorum sp. nov. Otra diferencia importante es que $A$. guatemalteca tiene una sola seda en los tubérculos dorsolaterales del tórax II y III, mientas que $A$. castagnorum posee 2 , una macroseda barbulada y una microseda barbulada (Cuadro 8).

Variación intraespecífica. Se compararon 4 machos y 1 juvenil; en la cabeza de este último fue posible observar un solo tubérculo Af que en los adultos parece haber desaparecido. En todas las muestras, el tubérculo DL del segmento abdominal IV se ve claramente separado y el número de sedas de los tubérculos DL $+\mathrm{L}$ presentan la siguiente variación: un ejemplar presenta en ambos tubérculos 2 macrosedas y 3 microsedas (2M, 3m) y respecto a éste, un ejemplar tiene $3 \mathrm{M}, 2 \mathrm{~m}$ y los otros ejemplares, MB, M.

\section{Americanura basseti sp. nov. (Figs. 14-15)}

Longitud $(\mathrm{n}=6) 0.79 \mathrm{~mm}$. Color blanco. Granulación cuticular fuerte. Tubérculos bien desarrollados. Con 4 tipos de sedas corporales, macrosedas barbuladas, (MB) $59.7 \mu \mathrm{m}(36.1$ - $86.4 \mu \mathrm{m})$, microsedas barbuladas (mb) 20.4 $\mu \mathrm{m}(14.1$ - $28.3 \mu \mathrm{m})$, microsedas lisas (m) $15.7 \mu \mathrm{m}(9.4$ $18.9 \mu \mathrm{m})$, macrosedas lisas (M) $40.8 \mu \mathrm{m}$ (31.4 - $50.3 \mu \mathrm{m})$, 
Cuadro 7. Quetotaxia total de Americanura castagnorum sp. nov.

\begin{tabular}{|c|c|c|c|c|}
\hline Grupo de sedas cefálicas & Tubérculos & Número de sedas & Tipo de sedas & Sedas \\
\hline $\mathrm{Cl}$ & 1 & 2 & $\mathrm{MB}, \mathrm{m}$ & $\mathrm{F}, \mathrm{G}$ \\
\hline Af & 1 & 1 & MB & B \\
\hline $\mathrm{Oc}$ & 1 & 1 & MB & Ocm \\
\hline $\mathrm{Di}$ & 1 & 1 & MB & Di1 \\
\hline De & 1 & 1 & $\mathrm{MB}$ & De1 \\
\hline $\mathrm{DL}+\mathrm{L}+\mathrm{So}$ & 1 & 9 & $2 \mathrm{MB}, 3 \mathrm{M}, 4 \mathrm{~m}$ & \\
\hline Suma total & 6 & 15 & & \\
\hline \multicolumn{5}{|c|}{ Grupo de sedas torácicas y abdominales } \\
\hline Tórax & & $D e$ & $D L$ & $L$ \\
\hline I & & $\mathrm{MB}$ & MB & - \\
\hline II & & $\mathrm{MB}+\mathrm{s}$ & $\mathrm{MB}, \mathrm{mb}+\mathrm{s}$ & $\mathrm{MB}, \mathrm{mb}, \mathrm{m}$ \\
\hline III & & $\mathrm{MB}+\mathrm{s}$ & $\mathrm{MB}, \mathrm{mb}+\mathrm{s}$ & $\mathrm{MB}, \mathrm{mb}$ \\
\hline \multicolumn{5}{|l|}{ Abdomen } \\
\hline I & & $\mathrm{MB}+\mathrm{s}$ & MB & $\mathrm{MB}, \mathrm{mb}, \mathrm{m}$ \\
\hline II & & $\mathrm{MB}+\mathrm{s}$ & $\mathrm{MB}$ & $\mathrm{MB}, \mathrm{mb}, \mathrm{m}$ \\
\hline III & & $\mathrm{MB}+\mathrm{s}$ & $2 \mathrm{MB}, 2 \mathrm{~m}$ & $\mathrm{MB}, 2 \mathrm{M}, 2 \mathrm{~m}$ \\
\hline IV & & $2 \mathrm{MB}+\mathrm{s}$ & & \\
\hline V & & $\mathrm{MB}+\mathrm{s}$ & $2 \mathrm{MB}$ & \\
\hline VI & & & & \\
\hline
\end{tabular}

Cuadro 8. Comparación de Americanura castagnorum sp. nov. y las especies más cercanas

\begin{tabular}{llllll}
\hline Especie & Cabeza & Di & De & DL & $L$ \\
\hline castagnorum sp nov. & $\mathrm{B}, \mathrm{F}, \mathrm{G}$ & $-11 / 11113$ & $111 / 11126$ & $123 / 1142$ & $-32 / 335$ \\
basseti sp. nov. & $\mathrm{B}, \mathrm{F}, \mathrm{G}$ & $-11 / 1111-$ & $112 / 22223$ & $133 / 2$ & $-11 / 2353$ \\
guatemalteca & $\mathrm{B}, \mathrm{F}, \mathrm{G}$ & $-11 / 11113$ & $111 / 1112$ & $111 / 111$ & $-33 / 2223$ \\
\hline
\end{tabular}

además de las sedas sensoriales (ss) $62.3 \mu \mathrm{m}(35-97.5 \mu \mathrm{m})$. Ant. I con 7 sedas, 3 son macrosedas. Ant. II con 11 sedas. Órgano sensorial del Ant. III con 2 sensilas globulares bajo un repliegue tegumentario, y 2 sensilas de guardia, la sensila de guardia ventral (sgv) ligeramente curva. Ant. IV de forma típica para el género con la sensila S7 hipertrofiada. Organito subapical ausente. Con $2+2$ ojos con pigmento negro. Mandíbulas con 3 dientes, maxilas estiletiformes. Cabeza con los tubérculos cefálicos: oculares, dorsointernos y (Di) y dorsoexternos (De) bien desarrollados. La Quetotaxia cefálica se muestra en el Cuadro 9 y se ilustra en la Fig. 14. Tibiotarsos I, II y III sin "tenent hair", con 18, 18 y 17 sedas respectivamente. Sin apéndice empodial. La quetotaxia torácica y abdominal se ilustra en las figuras 14 y 15, la quetotaxia total se muestra en el Cuadro 9. Tubo ventral con $4+4$ sedas basales, 3 pares dispuestos longitudinalmente subiguales al par distal que son más largas.

\section{Resumen taxonómico}

Material tipo. Holotipo hembra, 1 paratipo hembra, 1 paratipo macho, 2 paratipos hembra preadultas y 1 macho preadulto. Localidad tipo. PANAMÁ: Provincia Colón, San Lorenzo, bosque, 30.IX..2003; 4, 8, 11, 18.X.2003, N. Winchester y K. Jordan cols. dosel.

Etimología. Se nombra esta especie en honor a Yves Basset, coordinador científico del Programa del Dosel del Smithsonian Tropical Research Institute, Panamá, que ha contribuido al estudio de la fauna de la canopia.

\section{Comentarios taxonómicos}

Por la presencia de 2 sedas oculares, Americanura basseti sp. nov. es similar a $A$. setafoliacea (Cuadro 10); sin embargo, el tipo de sedas es muy diferente. Son foliadas 

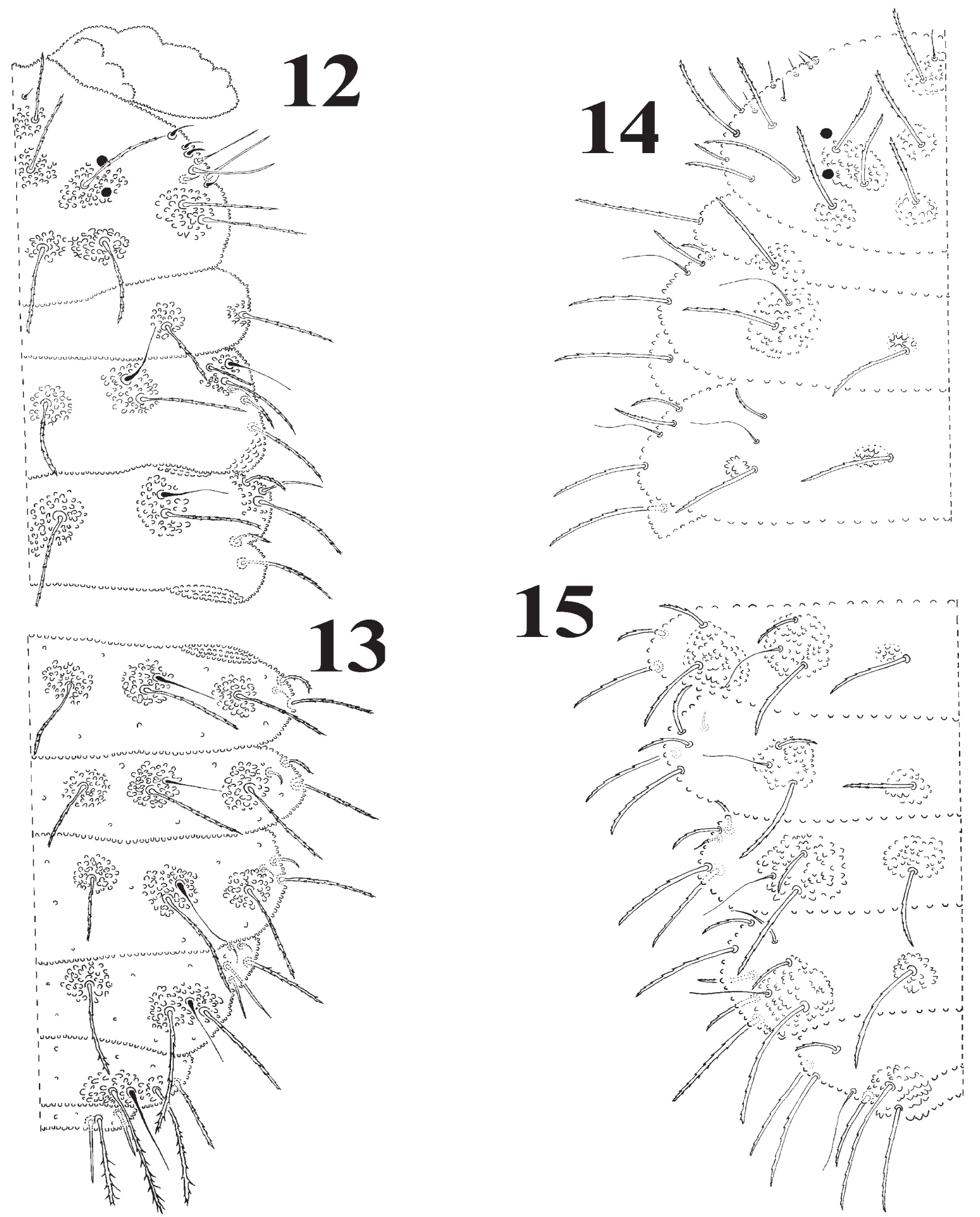

Figs. 12-15: 12 y 13, Americanura castagnorum sp. nov. 12, quetotaxia dorsal de la cabeza y segmentos torácicos I-III; 13, quetotaxia abdominal de los segmentos I-VI. 14 y 15, Americanura basseti sp. nov. 14, quetotaxia dorsal de la cabeza y segmentos torácicos I-III; 15 , quetotaxia abdominal de los segmentos I-VI. 
Cuadro 9. Quetotaxia total de Americanura basseti sp. nov.

\begin{tabular}{|c|c|c|c|c|}
\hline Grupo de sedas cefálicas & Tubérculos & Número de sedas & Tipo de sedas & Sedas \\
\hline $\mathrm{Cl}$ & 1 & 2 & $\mathrm{MB}, \mathrm{m}$ & $\mathrm{F}, \mathrm{G}$ \\
\hline Af & 1 & 1 & MB & $\mathrm{B}$ \\
\hline Oc & 1 & 2 & MB & Ocm, Ocp \\
\hline Di & 1 & 1 & MB & Di1 \\
\hline De & 1 & 1 & $\mathrm{MB}$ & De1 \\
\hline $\mathrm{DL}+\mathrm{L}+\mathrm{So}$ & - & - & & \\
\hline Suma total & 5 & 7 & & \\
\hline \multicolumn{5}{|c|}{ Grupo de sedas torácicas y abdominales } \\
\hline Tórax & $D i$ & $D e$ & $D L$ & $L$ \\
\hline I & - & MB & MB & - \\
\hline II & MB & $\mathrm{MB}+\mathrm{s}$ & $\mathrm{Mb}, 2 \mathrm{mb}+\mathrm{s}$ & MB \\
\hline III & MB & $\mathrm{MB}, \mathrm{mb}+\mathrm{s}$ & $\mathrm{MB}, 2 \mathrm{MB}+\mathrm{s}$ & MB \\
\hline \multicolumn{5}{|l|}{ Abdomen } \\
\hline I & MB & $\mathrm{MB}, \mathrm{mb}+\mathrm{s}$ & $\mathrm{MB}, \mathrm{mb}$ & $\mathrm{MB}, \mathrm{mb}$ \\
\hline II & MB & $\mathrm{MB}, \mathrm{mb}+\mathrm{s}$ & - & $2 \mathrm{MB}, \mathrm{mb}$ \\
\hline III & MB & $\mathrm{MB}, \mathrm{mb}+\mathrm{s}$ & - & $2 \mathrm{MB}, 2 \mathrm{mb}, \mathrm{m}$ \\
\hline IV & MB & $\mathrm{MB}, \mathrm{mb}+\mathrm{s}$ & - & $\mathrm{MB}, 2 \mathrm{mb}$ \\
\hline V & - & $2 \mathrm{MB}, \mathrm{mb}$ & & \\
\hline VI & $3 \mathrm{MB}+\mathrm{s}$ & & & \\
\hline
\end{tabular}

Cuadro 10. Comparación de Americanura basseti sp. nov. y las especies más cercanas

\begin{tabular}{llllll}
\hline Especie & Cabeza & Di & De & DL & $L$ \\
\hline basseti sp nov. & B, F, G & $-11 / 1111-$ & $112 / 22223$ & $133 / 2$ & $-11 / 2353$ \\
iztac sp nov. & C, D, F, G & $-33 / 222247$ & $222 / 2223$ & $133 / 222$ & $-33 / 3335$ \\
setafoliacea & B, F, G & $-11 / 111137$ & $111 / 1112$ & $111 / 111$ & $-33 / 2223$ \\
sotanophila & A, B, F, G & $-11 / 111137$ & $111 / 1111$ & $122 / 1111$ & $-33 / 3336$ \\
\hline
\end{tabular}

en la segunda especie y barbuladas en la primera. También parece cercana a $A$. sotanophila, pero la nueva especie tiene muy reducida la quetotaxia cefálica, lo que la aproxima más a $A$. basseti sp. nov. y A. guatemalteca de las cuales difiere por tener una quetotaxia mucho más abundante en los tubérculos dorsoexternos como se muestra en el Cuadro 8.

Variación intraespecifica. Se compararon 5 de 6 ejemplares; 2 adultos (hembra y macho) y 3 preadultos $(2$ hembras y 1 macho). La variación de las sedas de la cabeza es una constante en el grupo DL+L+So, 3 organismos presentaron un total de 10 sedas, mientras que los otros presentaron 6 y 7; el número de macrosedas barbuladas fue de 4, excepto en uno; en tanto que las microsedas son más en su número y tipo: $5 \mathrm{~m}, 5 \mathrm{mb}, 2 \mathrm{mb}, 3 \mathrm{mb}+2 \mathrm{~m}, 6 \mathrm{~m}$. En los segmentos abdominales IV, V y VI, 2 de 5 organismos presentaron variación de $3 \pm 1$ microseda barbulada $(\mathrm{mb})$ con respecto al patrón de comparación ( $\mathrm{MB}, \mathrm{M}, 3 \mathrm{mb}, \mathrm{m})$; en el tubérculo De+DL+L del segmento abdominal $\mathrm{V}$, un organismo tenía $3 \mathrm{mb}, 23 \mathrm{MB}$ y otros $2,4 \mathrm{MB}$, pero en estos últimos, sólo se presentó una microseda barbulada $(\mathrm{mb})$ adicional. Finalmente, en el tubérculo Di+De+DL+L del segmento abdominal VI, con respecto al patrón de comparación (2MB, 2M, 2mb), las microsedas barbuladas (mb) varían entre 1 y 3 , advirtiéndose la presencia de 1 sensila en aquellos con sólo 1 microseda barbulada; 2 de 5 organismos mostraron una variación de $2 \pm 1$ macroseda barbulada (MB), que con respecto al resto carecían de $2 \mathrm{M}$. 
Cuadro 11. Quetotaxia cefálica y longitud de las sedas en las nuevas especies de Americanura

\begin{tabular}{|c|c|c|c|c|c|c|c|c|c|c|c|c|c|c|c|c|}
\hline & \multirow{2}{*}{$\begin{array}{l}\text { Long. del } \\
\text { cuerpo (mm) }\end{array}$} & \multicolumn{3}{|c|}{ Long. de las sedas } & \multicolumn{2}{|c|}{$(\mu m)$} & \multicolumn{7}{|c|}{ Cefálicas } & \multicolumn{3}{|c|}{ Oculares } \\
\hline & & MB & $\mathrm{mb}$ & $\mathrm{m}$ & M & ss & A & B & $\mathrm{C}$ & $\mathrm{D}$ & $\mathrm{E}$ & F & G & Oca & $\mathrm{Ocm}$ & Ocp \\
\hline A. sardinasensis sp. nov. & 1.1 & 60 & 20 & 17 & 52 & 56 & - & + & + & - & - & + & + & + & + & + \\
\hline A. janssensi sp. nov. & 0.9 & 105 & 35 & 15 & 37 & 34 & + & + & - & - & - & + & + & - & + & + \\
\hline A. iztac sp. nov. & 1.6 & 107 & 45 & 30 & 65 & 42 & - & - & + & + & + & + & + & - & + & + \\
\hline A. castagnorum sp. nov. & 0.8 & 72 & 47 & 22 & 57 & 58 & - & + & - & - & - & + & + & - & + & - \\
\hline A. basseti sp. nov. & 0.8 & 60 & 20 & 15 & 40 & 62 & - & + & - & - & - & + & + & - & + & + \\
\hline
\end{tabular}

Presencia (+), ausencia (-) de sedas.

\section{Discusión}

De las 5 especies nuevas descritas, Americanura iztac sp. nov. es la de mayor tamaño, mientras que las 2 panameñas son las más pequeñas ( $A$. castagnorum sp. nov. y $A$. basseti sp. nov.); sin embargo, $A$. janssensi sp. nov. tiene macrosedas barbuladas relativamente largas. Las especies de Panamá son las más plesiomórficas en relación con la quetotaxia cefálica, ya que ambas sólo presentan las sedas B, F y G, no así $A$. castagnorum sp. nov., que presenta la apomorfia de haber perdido las sedas oculares Oca y Ocp, y tiene sólo una seda, la Ocm (Cuadro 11).

Es importarte resaltar que con estas 5 especies aumenta en $33 \%$ la riqueza de especies de este género que ya suman 20, convirtiéndose así en el género endémico de Neanurinae con mayor cantidad de especies del continente americano. Por otro lado, cabe señalar que tiene el $65 \%$ de las especies y que casi el $95 \%$ de todas las especies se ha encontrado en el Corredor Biológico Mesoamericano, y con el presente trabajo se extiende hasta Panamá la distribución del género.

\section{Agradecimientos}

Los ejemplares estudiados pertenecen a los proyectos: IN 223803 (Ecología de Ambientes Cavernícolas) del PAPIID, DGAPA, UNAM y el proyecto 33965-V (Ecología de una Cueva Sulfurosa) de CONACyT. Algunos ejemplares fueron proporcionados por Norma García Calderón, Gabriela Castaño Meneses, Leopoldo Cutz Pool y Arturo García Gómez. Blanca Estela Mejía Recamier colaboró en la preparación de los ejemplares para su estudio. Ada Alicia Ruiz Castillo ayudó en la elaboración de algunas figuras.

\section{Literatura citada}

Cassagnau, P. 1982. Sur les Neanurinea primitifs suceurs et les lignées qui en derivent (Collemboles). Travaux du Laboratoire d'Ecobiologie des Arthropodes Edaphiques, Toulouse 3:1-11.

Cassagnau, P. 1983. Un nouveau modèle phylogénétique chez les collemboles Neanurinae. Nouvelle Revue d'Entomologie 13:3-27.

Cassagnau, P. 1988. Les collemboles Neanurinae des massifs du sud de l'Inde et de Ceylan. Travaux du Laboratoire d'Ecobiologie des Arthropodes Edaphiques, Toulouse $5: 21-51$.

Deharveng, L. 1981. La chaetotaxie dorsal de l'antenne et son intérêt phylogenetique chez les collemboles Neanuridae. Nouvelle Revue d'Entomologie 11:3-13.

Deharveng, L. et W. M. Weiner. 1984. Collemboles de Corée du Nord. III-Morulinae et Neanurinae. Travaux du Laboratoire d'Ecobiologie des Arthropodes Edaphiques, Toulouse 4:1-64.

Palacios-Vargas, J. G. y J. C. Simón-Benito. 2007. Three new species of Nahuanura (Collembola: Neanuridae) from Mexico. Southwestern Entomologist 32:169-175. 